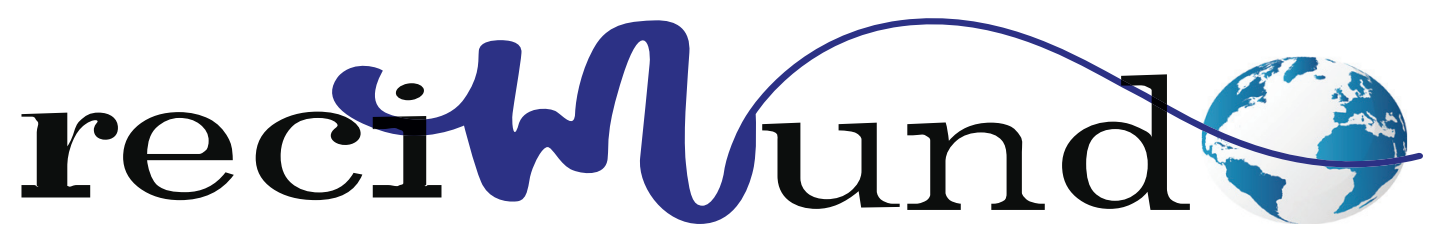

Revista Científica Mundo de la Investigación y el Conocimiento

DOI: 10.26820/recimundo/5.(2).abril.2021.140-148

URL: https://recimundo.com/index.php/es/article/view/1048

EDITORIAL: Saberes del Conocimiento

REVISTA: RECIMUNDO

ISSN: 2588-073X

TIPO DE INVESTIGACIÓN: Artículo de revisión

Código UNESCO: 32 Ciencias Médicas

PAGINAS: $140-148$

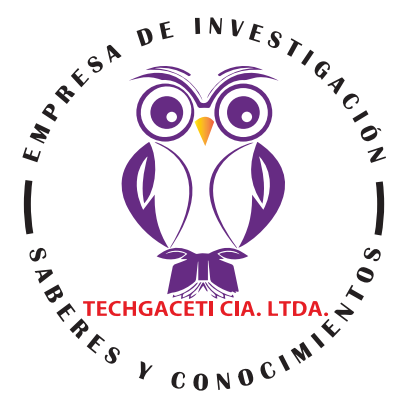

\title{
Anticoagulación vs profilaxis en COVID-19
}

Anticoagulation vs prophylaxis in COVID-19

Anticoagulação vs profilaxia na COVID-19

Amada Alaja García Bermúdes'; Luis Andrés Saldarriaga Zambrano²; Dennis Iván Narea Illescas3;

Josselin Fabiola Chávez Almeida ${ }^{4}$

RECIBIDO: 15/01/2021 ACEPTADO: 20/03/2021 PUBLICADO: 01/04/2021

1. Médico de la Universidad Católica de Santiago de Guayaquil; Investigador Independiente; Guayaquil, Ecuador; amaditagb23@gmail.com; iD https://orcid.org/0000-0002-4117-8206

2. Médico de la Universidad de Guayaquil, Investigador Independiente; Guayaquil, Ecuador; I.andres1990@hotmail.com; https://orcid.org/0000-0002-1872-079X

3. Médico de la Universidad de Guayaquil, Investigador Independiente; Guayaquil, Ecuador; ivan-2109@hotmail.com; https://orcid.org/0000-0001-8292-0975

4. Médico de la Universidad de Guayaquil, Investigador Independiente; Guayaquil, Ecuador; drajosselinchavez@gmail.com;

(iD) https://orcid.org/0000-0003-0792-5076

\section{CORRESPONDENCIA}

Amada Alaja García Bermúdes

amaditagb23@gmail.com

Guayaquil, Ecuador

(c) RECIMUNDO; Editorial Saberes del Conocimiento, 2021 


\section{RESUMEN}

La infección por SARS-CoV-2 puede desencadenar una cascada inflamatoria que se acompaña de un aumento del riesgo de enfermedad tromboembólica. Por esa razón distintos consensos recomiendan la anticoagulación, al menos profiláctica. La sociedad internacional de trombosis y hemostasia (ISTH) define CID como un síndrome adquirido que se caracteriza por la activación intravascular de la coagulación con lo cual se desencadena la producción de grandes cantidades de trombina y fibrina intravascular que puede, a su vez, dañar la microvasculatura y generar disfunción multiorgánica. Es importante destacar que algunas de estas alteraciones se han relacionado con un peor pronóstico, sobre todo el incremento del DD. Así, los pacientes con infección COVID 19 grave, especialmente los ingresados en Unidades de Cuidados Críticos, presentan un riesgo particularmente elevado de desarrollo de complicaciones cardiovasculares, en particular enfermedad tromboembólica venosa. En estas circunstancias, es importante establecer la terapia anticoagulante profiláctica o terapéutica que pueda beneficiar a estos pacientes. La metodología de la investigación, es una revisión bibliográfica, apoyada en medios electrónicos como fuente primaria de obtención de la información. Entre las conclusiones se pueden mencionar que a pesar de las contraindicaciones o efectos secundarios que puede generar el uso de la hidroxicloroquina y cloroquina como tratamiento profiláctico, pareciera ser más efectivo que el tratamiento anticoagulante. Sin embargo, toda evolución del paciente va a depender del rápido diagnóstico de las complicaciones que se generen y su tratamiento.

Palabras clave: Profiláctico, Coagulación, Covid 19, Dimero D, Cloroquina.

\section{ABSTRACT}

SARS-CoV-2 infection can trigger an inflammatory cascade that is accompanied by an increased risk of thromboembolic disease. For this reason, different consensuses recommend anticoagulation, at least prophylactic. The International Society for Thrombosis and Haemostasis (ISTH) defines DIC as an acquired syndrome characterized by intravascular activation of coagulation, which triggers the production of large amounts of intravascular thrombin and fibrin, which can, in turn, damage the blood vessels. microvasculature and generate multiorgan dysfunction. It is important to highlight that some of these alterations have been related to a worse prognosis, especially the increase in DD. Thus, patients with severe COVID 19 infection, especially those admitted to Critical Care Units, present a particularly high risk of developing cardiovascular complications, particularly venous thromboembolic disease. In these circumstances, it is important to establish prophylactic or therapeutic anticoagulant therapy that can benefit these patients. The research methodology is a bibliographic review, supported by electronic media as the primary source for obtaining information. Among the conclusions it can be mentioned that despite the contraindications or side effects that the use of hydroxychloroquine and chloroquine as prophylactic treatment can generate, it seems to be more effective than anticoagulant treatment. However, all evolution of the patient will depend on the rapid diagnosis of the complications that are generated and their treatment.

Keywords: Prophylactic, Coagulation, Covid 19, Dimero D, Chloroquine.

\section{RESUMO}

A infecção pelo SARS-CoV-2 pode desencadear uma cascata inflamatória que é acompanhada por um aumento do risco de doença tromboembólica. Por esta razão, diferentes consensos recomendam a anticoagulação, pelo menos profilática. A Sociedade Internacional para Trombose e Hemostasia (ISTH) define DIC como uma síndrome adquirida caracterizada pela ativação intravascular da coagulação, que desencadeia a produção de grandes quantidades de trombina e fibrina intravascular, que podem, por sua vez, danificar os vasos sanguíneos. microvasculatura e gerar disfunção multiorgânica. É importante destacar que algumas destas alterações têm sido relacionadas a um pior prognóstico, especialmente o aumento da DD. Assim, pacientes com infecção grave pela COVID 19, especialmente aqueles admitidos em Unidades de Cuidados Críticos, apresentam um risco particularmente alto de desenvolver complicações cardiovasculares, particularmente a doença tromboembólica venosa. Nessas circunstâncias, é importante estabelecer uma terapia profilática ou terapêutica anticoagulante que possa beneficiar esses pacientes. A metodologia de pesquisa é uma revisão bibliográfica, apoiada por meios eletrônicos como a principal fonte para a obtenção de informações. Dentre as conclusões, pode-se mencionar que apesar das contra-indicações ou efeitos colaterais que o uso de hidroxicloroquina e cloroquina como tratamento profilático pode gerar, parece ser mais eficaz do que o tratamento anticoagulante. Entretanto, toda a evolução do paciente dependerá do rápido diagnóstico das complicações que forem geradas e de seu tratamento.

Palavras-chave: Profilático, Coagulação, Covid 19, Dimero D, Cloroquina. 

J. F.

\section{Introducción}

La infección por SARS-CoV-2 puede desencadenar una cascada inflamatoria que se acompaña de un aumento del riesgo de enfermedad tromboembólica. Por esa razón distintos consensos recomiendan la anticoagulación, al menos profiláctica (Álvarez-Rodríguez, y otros, 2020).

Entre las principales complicaciones de esta enfermedad están los fenómenos hematológicos, destaca la coagulopatía, particularmente coagulación intravascular diseminada con fenotipo protrombótico que se asocia con mal pronóstico. Los fenómenos trombóticos en enfermedad severa se han descrito, según la serie, en $8 \%$ a más de $30 \%$ de los pacientes (Jiménez-Soto, Montiel-Romero, Neme-Yunes, Demichelis-Gómez, \& Inclán-Alarcón, 2020, pág. 211).

La sociedad internacional de trombosis y hemostasia (ISTH) define CID como un síndrome adquirido que se caracteriza por la activación intravascular de la coagulación con lo cual se desencadena la producción de grandes cantidades de trombina y fibrina intravascular que puede, a su vez, dañar la microvasculatura y generar disfunción multiorgánica (Abuabara-Franco, y otros, 2020).

La infección por coronavirus tipo COVID-19 se asocia con frecuencia a alteraciones de determinados parámetros de la coagulación. El patrón típico incluye las siguientes características:

- incremento significativo en el nivel plasmático del dímero D (DD)

- incremento significativo en el nivel plasmático de fibrinógeno (FIB)

- alargamiento moderado del tiempo de protrombina (TP)

- normalidad en el tiempo parcial de tromboplastina activada (TPTa)

- tendencia a trombocitosis moderada

- disminución moderada, no clínicamente significativa, de la actividad de antitrombina (AT).

Es importante destacar que algunas de estas alteraciones se han relacionado con un peor pronóstico, sobre todo el incremento del DD. Así, los pacientes con infección COVID 19 grave, especialmente los ingresados en Unidades de Cuidados Críticos, presentan un riesgo particularmente elevado de desarrollo de complicaciones cardiovasculares, en particular enfermedad tromboembólica venosa. En estas circunstancias, es importante establecer la terapia anticoagulante profiláctica o terapéutica que pueda beneficiar a estos pacientes (Llau, y otros, 2020, pág. 1).

Uno de los mecanismos que distingue una infección por SARS-Cov-2 de otras coagulopatías comúnmente observadas en pacientes con infección grave, es el daño directo a las células endoteliales. Esto da lugar a una liberación masiva de componentes celulares endoteliales, como los multímeros del FVW y los activadores del plasminógeno. En pacientes con inflamación sistémica, los niveles de ADAMTS-13 están descendidos, por lo que no pueden degradarse de una manera proporcional los multímeros generados del FVW. Esta acumulación de multímeros induce a la trombosis microvascular. Por otra parte, la liberación del activador de plasminógeno da como resultado una generación de plasmina y puede explicar el aumento excesivo de los niveles de D-dimero (Vidal, 2020, pág. 70).

La prescripción de medicamentos para COVID-19 debe realizarse bajo indicaciones médicas muy precisas y no debe usarse en profilaxis. Sin embargo, existen estudios de Investigación en curso para establecer su rol de profilaxis en los trabajadores de la salud (Zapata, 2020).

Por múltiples debates médicos, políticos y sociales, que en algunos contextos no tienen base científica, existe evidencia cre- 
ciente que recomienda el uso de cloroquina o hidroxicloroquina como profilaxis en algunas poblaciones expuestas de riesgo como profesionales de salud y contactos estrechos de pacientes con COVID19, lo que ha llevado al desarrollo de múltiples ensayos clínicos con la intención de responder a la pregunta sobre si las dosis profilácticas de hidroxicloroquina o cloroquina pueden disminuir el riesgo de infección clínica en personas con alta exposición a COVID-19 (Zapata, 2020).

Profilaxis pre-exposición: Indicación: Personal de la salud, asintomático, que trabaja en áreas de atención de una alta carga de casos sospechosos o confirmados de COVID-19, o con alto riesgo de una exposición inadvertida.

- $1^{\circ}$ opción: HCLQ 400 mg cada 12 horas el primer día y luego 400 mg cada semana vía oral hasta finalizar la exposición

- $2^{\circ}$ opción: CLQ 250 mg cada 12 horas vía oral por máximo 3 meses (Zapata, 2020).

Profilaxis post-exposición: Indicación: Personal de la salud, asintomático, que ha tenido un contacto estrecho con un caso sospechoso o confirmado de COVID-19.

- $\quad 1^{\circ}$ opción: HCLQ 200 mg cada 12 horas vía oral por 5 días

- $2^{\circ}$ opción: CLQ 500 mg cada 12 horas vía oral por 5 días (Zapata, 2020).

Estudios muestran la posible inhibición del receptor ECA2, mediante el uso de la Cloroquina/Hidroxicloroquina, pues interfiere con su glicosilación terminal; por lo que este fármaco podría actuar en varios pasos del ciclo de replicación viral del SARS-COV-2. En febrero de 2020, se demostró que ambos medicamentos reducen efectivamente la enfermedad de la COVID-19, pero un estudio adicional concluyó que la Hidroxicloroquina era más potente que la Cloroquina y tenía un perfil de seguridad más tolerable y seguro en la neumonía por la COVID-19, al mejorar los hallazgos de las imágenes pulmonares, promover una conversión negativa al virus y acortar el curso de la enfermedad (Gómez Tejeda, Dieguez Guach, \& Pérez Abreu, 2020).

\section{Metodología}

La metodología de la investigación, es una revisión bibliográfica que se apoyó en medios electrónicos como Google académico, PubMed, entre otros. Donde se encontró información relevante que ayudo a la consecución del presente trabajo. El objetivo fundamental es crear un instrumento de trabajo bastante explicito y completo que permita ser una referencia para otros investigadores, acerca del tema de estudio.

\section{Resultados}

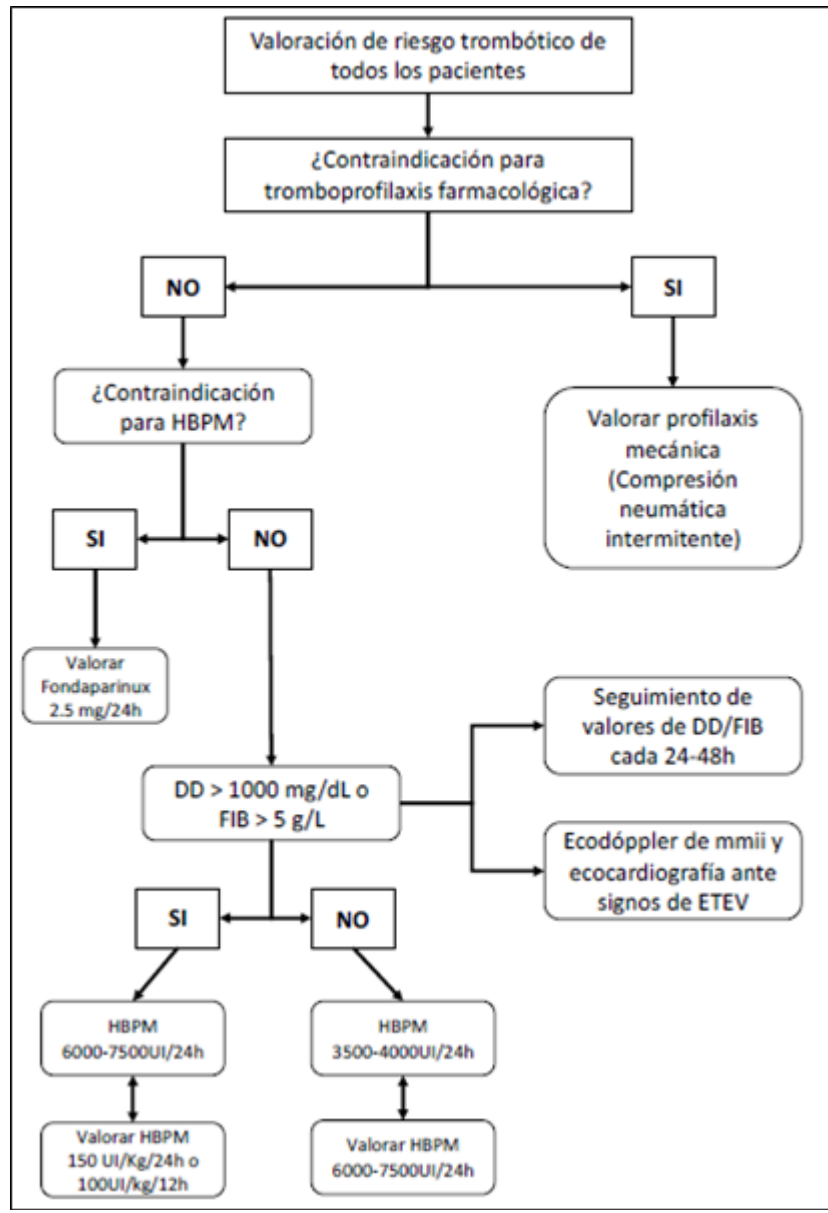

Imagen I. Tromboprofilaxis en pacientes con infección por covid-19 ingresados en

las unidades de cuidados intensivos. 

J. F.

Esta coagulopatía se manifestaría con elevación del dímero $D$ y de los productos de degradación de fibrina/fibrinógeno, mientras que las alteraciones en el tiempo de protrombina, el tiempo de tromboplastina parcial activado y el recuento de plaquetas son relativamente poco comunes al inicio del cuadro. Sin embargo, aunque el aumento de dímero $D$, las manifestaciones de sepsis y la coagulopatía de consumo son indicadores de mortalidad, los datos actuales no sugieren el uso de anticoagulación a dosis terapéuticas en todos los pacientes COVID-19, a menos que esté clínicamente indicado, lo cual ha generado controversia ya que otros estudios observacionales describen mejor pronóstico en pacientes con COVID-19 grave tratados con anticoagulantes orales por heparina de bajo peso molecular (HBPM) (Álvarez-Rodríguez, y otros, 2020).

- En los pacientes, con infección grave por Covid-19, ingresados en las plantas de hospitalización, se recomienda la administración de tromboprofilaxis farmacológica, siendo de elección la heparina de bajo peso molecular, a las dosis habituales (3500-4000 Ul/24h).

- En los casos con antecedentes de trombocitopenia inducida por heparina $(\mathrm{TIH})$, se sugiere plantear como alternativa la administración de fondaparinux (2.5 mg/24h sc) (fuera de indicación).

- En los pacientes graves ingresados en las Unidades de Cuidados Críticos y/o siempre que el nivel plasmático de algunos marcadores (DD, FIB), se encuentren claramente elevados respecto a valores de referencia, se recomienda incrementar la dosis de HBPM a dosis intermedias (6000-7500 UI/24h, según peso).

- Con los datos actuales no se puede recomendar dosis de anticoagulación terapéutica (10.000 Ul/24h, $150 \mathrm{Ul} / \mathrm{kg} / 24 \mathrm{~h}$ o $100 \mathrm{UI} / \mathrm{kg} / 12 \mathrm{~h}$ ) a todos los pacientes. Sin embargo, se sugiere valorar esta dosis, si a la elevación de marcadores (ya comentada), se asocian trombocitosis (> 5x109/L) y/o hiperfibrinogenemia (> $5 \mathrm{~g} / \mathrm{L})$.

- Se recomienda el ajuste de dosis en función del aclaramiento de creatinina $(\mathrm{ClCr}<30 \mathrm{ml} / \mathrm{min}$ ) y la monitorización de los niveles de anti-Xa en los casos de insuficiencia renal. En el caso de disponer de tinzaparina, se sugiere utilizar ésta a dosis equivalentes.

- Se recomienda el seguimiento analítico de los valores de DD, fibrinógeno, TP, recuento de plaquetas, así como la realización de la puntuación de CID, según protocolo establecido en cada centro (a intervalos de 24-72h como máximo).

- Se recomienda considerar la posibilidad de tromboembolismo pulmonar en los pacientes con deterioro súbito de la oxigenación, distress respiratorio y caída brusca de la presión arterial.

- En los casos de alto riesgo hemorrágico, la tromboprofilaxis se realizará preferentemente con métodos mecánicos (compresión neumática intermitente). En estas circunstancias, se sugiere valorar la realización de test viscoelásticos como complemento de la monitorización de la hemostasia (Llau, y otros, 2020).

Se ha documentado que los pacientes con esta infección están predispuestos a tromboembolismo venoso y arterial por mecanismos de inflamación excesiva, hipoxemia, inmovilización y por la existencia de coagulación intravascular diseminada, esta última es prevalente en, incluso, $71 \%$ de los pacientes que fallecieron por esta enfermedad, de acuerdo con los criterios de la Sociedad Internacional de Trombosis y Hemostasia (ISTH por sus siglas en inglés) (Jiménez-Soto, Montiel-Romero, Neme-Yunes, Demichelis-Gómez, \& Inclán-Alarcón, 2020).

La hipoxemia se genera por diversos mecanismos, como daño alveolar directo y trombosis. En una neumonía severa existe un estado procoagulante, no solo por el in- 
cremento de la viscosidad sanguínea, sino también por la señalización de la transcripción inducida por hipoxia, perpetuando la relación hipoxemia-trombosis (Jiménez-Soto, Montiel-Romero, Neme-Yunes, Demichelis-Gómez, \& Inclán-Alarcón, 2020).

La circulación libre de trombina, no controlada por los anticoagulantes naturales, puede activar las plaquetas y estimular la fibrinólisis. Según el estudio, en las últimas etapas de la neumonía por el nuevo Coronavirus, los niveles de marcadores de fibrina (dímero D y FDP) se elevaron moderada o notablemente en todas las muertes, lo que sugirió una activación de la coagulación y una condición secundaria de hiperfribinolisis en estos pacientes. Se considerarán factores de mayor riesgo trombótico en los pacientes con la COVID-19, las formas graves con las siguientes alteraciones: proteína $\mathrm{C}$ reactiva $>150 \mathrm{mg} / \mathrm{L}$, dímero $\mathrm{D}>1500 \mathrm{ng} / \mathrm{mL}$, ferritina $>1000 \mathrm{ng} / \mathrm{mL}$, linfocitopenia $<800 \times 10^{\wedge} 6 / \mathrm{L}$, IL-6 >40 pg/mL y dímero-D >3000 ng/ml. Además, antecedentes personales o familiares de enfermedad trombótica venosa y antecedentes personales de enfermedad trombótica arterial. Trombofilia biológica conocida, cirugía reciente, gestación y terapia hormonal sustitutiva (Gómez Tejeda, Dieguez Guach, \& Pérez Abreu, 2020).

En todos pacientes hospitalizados en sala general y unidad de cuidados intensivos, en ausencia de complicaciones tromboembólicas y contraindicaciones para la terapia, se recomienda la tromboprofilaxis con heparina de bajo peso molecular, heparina no fraccionada y el fondaparinux. Cabe resaltar, que existe preferencia sobre la heparina de bajo peso molecular y la heparina no fraccionada frente el fondaparinux, sobre todo en el paciente crítico. No se recomienda el manejo antiplaquetario como prevención de TEV (Abuabara-Franco, y otros, 2020).

En los casos de diagnóstico positivo para TVP/ EP en pacientes en hospitalización general o UCI, sin choque o falla cardíaca de- recha, se sugiere la anticoagulación parenteral con heparina de bajo peso molecular o heparina no fraccionada. Los pacientes con complicaciones tromboembólicas asociadas a choque o falla cardiaca derecha, se recomienda la trombolisis sistémica. En la administración de la terapia trombolítica se prefiere el uso de un acceso periférico frente al acceso central (Abuabara-Franco, y otros, 2020).

\section{Profilaxis}

La CLQ es un agente antimalárico usado por más de 70 años, de fácil acceso, con propiedades comprobadas de quimioprofilaxis en malaria. Se sabe que el SARS-CoV-2 se une a las células humanas a través del receptor de la enzima convertidora de angiotensina 2 (ECA 2). Los estudios in vitro han demostrado que el proceso de glicosilación del receptor ECA 2 se ve afectado, lo que hace que las células Vero pretratadas in vitro con CLQ sean refractarias a la infección por COVID-19, que puede ser el mecanismo a través del cual incluso las células humanas pueden volverse refractarias a esta infección. También se ha visto que el tratamiento con CLQ previene la propagación de la infección por COVID-19 en el período posterior a la infección (Zapata, 2020).

Datos preliminares de algunos ensayos clínicos en China han demostrado que la CLQ podría reducir la duración de la estancia hospitalaria y mejorar la evolución de la neumonía por COVID-19, lo que ha llevado a recomendar la administración de 500 mg de cloroquina dos veces al día en pacientes con formas leves, moderadas y severas de neumonía por COVID-19. La dosis óptima para el COVID-19 aún es motivo de controversia y estudio; la actividad de la HCLQ en los virus es probablemente la misma que la de la CLQ, por tener el mismo mecanismo de acción, pero con un mejor perfil de seguridad, convirtiendo la HCLQ en la primera opción en el tratamiento de COVID-19. Para el tratamiento puede ser necesario adminis- 

J. F.

trar una dosis de carga seguida de una dosis de mantenimiento (Zapata, 2020).

La HCLQ ha mostrado actividad antiviral in vitro contra SARS-CoV-2. El modelo farmacológico basado en las concentraciones observadas de la HCLQ in vitro sugiere que la profilaxis con HCLQ a dosis aprobadas para uso en humanos podría prevenir la infección por SARS-CoV-2 y mejorar la eliminación viral (Zapata, 2020).

Se ha mencionado aparte de su efecto antipalúdico, por parte de la Cloroquina otros efectos potenciales como efectos antivirales (de ahí su uso en algunas enfermedades de este tipo), y como droga modificadora de enfermedades reumatológicas; además de haber sido mencionada como un potente inhibidor de SARS-CoV por interferir con el ACE2, que es el sitio de anclaje de la proteína S del SARS-CoV; el uso de CQ e HCQ se fundamenta en la afinidad competitiva de los fármacos con el punto de anclaje viral por parte del SARS-CoV-2 de estas con el receptor de la enzima convertidora de angiotensina 2 (similar al SARS-CoV), ya que como se ha mencionado es el único receptor de entrada celular (ya que no detectaron el uso de otros receptores de entrada a la célula que habitualmente usan los coronavirus, tales como aminopeptidasa $\mathrm{N}$ y dipeptidil peptidasa); así, el bloqueo de la unión de la proteína S a ACE2 es considerado un factor clave para el desarrollo de estudios de tratamiento de la infección por SARSCoV (Rojas, y otros, 2020).

Tanto la CQ como la HCQ han sido mencionadas como agentes eficientes para lograr una impronta en la mejoría clínica del COVID-19 y un aclaramiento viral más rápido de SARS-CoV-2; las dosis experimentales usadas de HCQ son $1200 \mathrm{mg}$ como dosis máxima tolerable y para CQ 500 mg., respectivamente (Rojas, y otros, 2020).

Hasta el momento no existe evidencia que respalde el uso de $\mathrm{CQ} / \mathrm{HCQ}$ en la profilaxis del SARSCoV-2, pues la información disponible sobre su eficacia clínica, sola y combinada con azitromicina, se basa principalmente en análisis preliminares de series retrospectivas, estudios de cohorte u observacionales y ensayos clínicos con limitaciones metodológicas que impiden generalizar los resultados. Por tanto, su uso debe ser cauteloso y basado en el principio de prevención, pues además las últimas publicaciones sugieren cardiotoxicidad (Acevedo, Pinzón, Barrera, Padilla, \& González, 2020).

\section{Conclusiones}

Existe una controversia por la bibliografía revisada, acerca de la utilización de la profilaxis como tratamiento en pacientes con Covid 19, así como su utilización como medida preventiva para evitar el contagio en personal de alto riesgo que atiende a pacientes infectados, así como el resto del personal de salud. Sin embargo, hay diferentes estudios que si aprueban su uso como medida de combate a la enfermedad ya que los mismos actúan como un efecto antiviral que detiene la propagación del virus en el organismo, por su efecto en la inhibición de la replicación de las células Vero, en cambio los detractores de el uso de la Cloroquina y la Hidroxicloroquina como tratamiento ante el Covid, argumentan sobre los efectos secundarios que pueden causar en el organismo, ya que las altas dosis de estos medicamentos pueden aumentar la mortalidad y requerimiento de ventilación mecánica, arritmias ventriculares, función hepática y renal, entre otros.

Existen estudios que en fases avanzadas de Covid 19, donde hay riesgo de coagulación intravascular diseminada con un fenotipo predominantemente trombótico. A pesar que en múltiples trabajos de investigación colocan las dosis recomendadas para su tratamiento, sin embargo, no hay una guía que especifique realmente cuanto es la dosis intermedia o terapéutica recomendada. Hay otros estudios que indican que se debe 
recomendar la anticoagulación a dosis profiláctica en la mayoría de los pacientes, sin embargo, no sucede los mismo con la anticoagulación terapéutica en pacientes graves, ya que puede haber riesgos.

En líneas generales en la bibliografía revisada no existe evidencia en cuanto a la profilaxis, de procesos de coagulación, solo se hace mención de los beneficios que tanto la hidroxicloroquina como la cloroquina pueden tener en la contención y destrucción del avance del Covid 19 en el cuerpo, lo que hace deducir que también son efectivos en el proceso de coagulación, ya que, si detienen el avance del virus, pueden detener todas las complicaciones asociadas al mismo. Se sabe que hasta el $71 \%$ de los pacientes fallecidos por Covid 19 estuvieron predispuestos a tromboembolismo venoso y arterial por mecanismos de inflamación excesiva, hipoxemia, inmovilización y por la existencia de coagulación intravascular diseminada. La efectividad del tratamiento esta asociada a su inicio temprano en pacientes que no estén graves, ya que en pacientes muy graves el pronostico de muerte es elevado, si el proceso de coagulación no es atacado a tiempo, la probabilidad de muerte se puede dar entre los 4 - 11 días desde el inicio de la misma.

Como conclusión general a pesar de las contraindicaciones o efectos secundarios que puede generar el uso de la hidroxicloroquina y cloroquina, pareciera ser mas efectivo este tratamiento que el anticoagulante. Sin embargo, toda evolución del paciente va a depender del rápido diagnóstico de las complicaciones que se generen y su tratamiento.

\section{Bibliografía}

Abuabara-Franco, E., Serpa-Díaz, D., Leal-Martínez, V., Pájaro-Galvis, N., Correa-Guerrero, J., Rico-Fontalvo, J., \& Fontalvo, J. (2020). Anticoagulación en pacientes con infección por SARS-CoV-2/ COVID-19. Salud UIS, 52(4).

Acevedo, K. C., Pinzón, C., Barrera, A., Padilla, E., \& González, N. (2020). Eficacia y seguridad de la cloroquina, la hidroxicloroquina y la azitromicina en pacientes con COVID-19. Resumen de evidencia. Revista Colombiana de Nefrología, 7(2). doi:http://dx.doi.org/10.22265/acnef.7.Supl.2.469

Álvarez-Rodríguez, E., González González, R., Torres-Gárate, R., López Riquelme, P., González Martil, I., \& Abad Cuñado, V. (2020). Aprendiendo con el COVID-19: ¿ qué pasa con la anticoagulación? In Anales del Sistema Sanitario de Navarra. Gobierno de Navarra. Departamento de Salud, 43(2), 251-254. doi:https://doi.org/10.23938/ASSN.0884

Gómez Tejeda, J. J., Dieguez Guach, R., \& Pérez Abreu, M. (2020). Alternativas terapéuticas para el manejo de la COVID-19. Revista Habanera de Ciencias Médicas, 19. Obtenido de http:// www.revhabanera.sld.cu/index.php/rhab/article/ view/3328

Jiménez-Soto, R., Montiel-Romero, S., Neme-Yunes, Y., Demichelis-Gómez, M., \& Inclán-Alarcón, S. (2020). Estrategias de anticoagulación en pacientes con COVID-19. Revista de Hematología, 21(4), 210-224.

Llau, J. V., Ferrandis, R., Sierra, P., Hidalgo, F., Cassinello, C., Gómez-Luque, A., \& la Victoria, M. (2020). Propuesta de recomendaciones de manejo de fármacos anticoagulantes y antiagregantes en los pacientes graves con infección por COVID-19. Obtenido de https://www. sedar. es/images/site/ NOTICIAS/coronavirus/RECOMENDACIONES_hemostasia-COVID-final. pdf.

Rojas, G. A., Bayona, D., Bayona, C., Cano, H., Cano, G., \& Rojas, K. (2020). ¿ Profilaxis con Cloroquina y/o Hidroxicloroquina para COVID-19? INVESTIGATIO, 15, 1-10.

Vidal, J. B. (2020). Alteraciones hematológicas y COVID-19. Medicina balear, 35(4), 69-73. doi:10.3306/ MEDICINABALEAR.35.04.69

Zapata, N. D. (2020). USO DE PROFILAXIS CONTRA COVID-19: Revisión de la Evidencia. 

J. F.

\section{CITAR ESTE ARTICULO:}

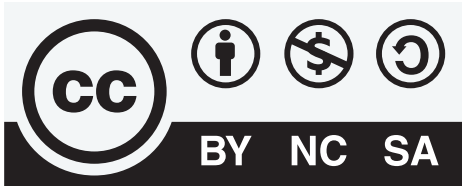

CREATIVE COMMONS RECONOCIMIENTO-NOCOMERCIAL-COMPARTIRIGUAL 4.0 .

García Bermúdes, A. A., Saldarriaga Zambrano, L. A., Narea Illescas, D. I., \& Chávez Almeida, J. F. (2021). Anticoagulación vs profilaxis en COVID-19. RECIMUNDO, 5(2), 140-148. https://doi.org/10.26820/recimundo/5.(2). abril.2021.140-148 